\title{
Triple A patient cells suffering from mitotic defects fail to localize PGRMC1 to mitotic kinetochore fibers
}

\author{
Ramona Jühlen ${ }^{1,2^{*}} \mathbb{D}$, Dana Landgraf ${ }^{1}$, Angela Huebner ${ }^{1}$ and Katrin Koehler ${ }^{*}$
}

\begin{abstract}
Background: Membrane-associated progesterone receptors are restricted to the endoplasmic reticulum and are shown to regulate the activity of cytochrome P450 enzymes which are involved in steroidogenesis or drug detoxification. PGRMC1 and PGRMC2 belong to the membrane-associated progesterone receptor family and are of interest due to their suspected role during cell cycle. PGRMC1 and PGRMC2 are thought to bind to each other; thereby suppressing entry into mitosis. We could previously report that PGRMC2 interacts with the nucleoporin ALADIN which when mutated results in the autosomal recessive disorder triple A syndrome. ALADIN is a novel regulator of mitotic controller Aurora kinase $A$ and depletion of this nucleoporin leads to microtubule instability.

Results: In the current study, we present that proliferation is decreased when ALADIN, PGRMC1 or PGRMC2 are overexpressed. Furthermore, we find that depletion of ALADIN results in mislocalization of Aurora kinase A and PGRMC1 in metaphase cells. Additionally, PGRMC2 is over-expressed in triple A patient fibroblasts.

Conclusion: Our results emphasize the possibility that loss of the regulatory association between ALADIN and PGRMC2 gives rise to a depletion of PGRMC1 at kinetochore fibers. This observation may explain part of the symptoms seen in triple A syndrome patients.
\end{abstract}

Keywords: ALADIN, Kinetochore fibers, Mitosis, Nuclear pore complex, PGRMC1, PGRMC2, Triple A syndrome

\section{Background}

ALADIN is a scaffold nucleoporin (NUP) anchored within the nuclear pore complex by the transmembrane NUP NDC1 [1, 2]. ALADIN seems to be involved in building the structural scaffold backbone of the nuclear pore complex [3]. Over the last years it has been shown that nuclear pore complexes and its NUPs have fundamental functions beyond nucleocytoplasmic transport and control cellular dysfunction in a variety of cellular pathways, especially during mitosis [4-6]. The first report that ALADIN plays a role during cell division

\footnotetext{
*Correspondence: rjuhlen@ulb.ac.be; katrin.

koehler@uniklinikum-dresden.de

${ }^{1}$ Klinik und Poliklinik für Kinder- und Jugendmedizin, Medizinische

Fakultät Carl Gustav Carus, Technische Universität Dresden,

01307 Dresden, Germany

${ }^{2}$ Present Address: Institute for Molecular Biology and Medicine, Université

Libre de Bruxelles, 6041 Charleroi, Belgium
}

was published in 2015 [7]. In cooperation with Carvalhal et al. we proposed ALADIN as novel regulator of mitotic kinase Aurora kinase A and showed that depletion of the nucleoporin resulted in impaired mitotic spindle assembly and chromosomal alignment at the metaphase plate [7]. Furthermore, we could recently document that ALADIN is necessary for murine oocyte maturation and for specific stages during meiosis [8].

Mutations in the human $A A A S$ gene, coding for the protein ALADIN (alacrima-achalasia-adrenal insufficiency neurologic disorder), lead to the autosomal recessive disorder named triple A syndrome $[9,10]$. Triple A patients present with three distinct symptoms: absent adrenal glucocorticoid and mineralocorticoid synthesis (adrenal insufficiency), impaired movement of the stomach cardia (achalasia) and loss of tear production (alacrima) [11]. These symptoms are highly heterogeneous and are accompanied by progressive 
impairments of the central, peripheral or autonomous nervous system [11]. Most mutations in AAAS result in a mis-localization of ALADIN to the cytoplasm [12, 13].

Previously, we identified PGRMC2 as novel interactor for the nucleoporin ALADIN and provided new insights into the molecular function of the nucleoporin in the pathogenesis of triple A syndrome [14]. PGRMC2 belongs to the group of membrane-associated progesterone receptors. These receptors mainly localize to the endoplasmic reticulum (ER) and are thought to regulate the activity of microsomal cytochrome (CYP) P450 enzymes which are involved in steroidogenesis or drug detoxification [15]. The first identified membraneassociated progesterone receptor, PGRMC1, gained wide-spread attention due to its several implications in cancerogenesis [16-19]. The mixed-function oxidase system of CYP P450 enzymes requires a donor transferring electrons from NADPH to reduce the enzyme's prosthetic heme group [20]. PGRMC1 and PGRMC2 contain a CYP b5-similar heme-binding domain which makes them possible electron donors for CYP P450 enzymes. [19]. Indeed, PGRMC1 forms stable proteinprotein complexes with CYP51A1, CYP7A1, CYP21A2 and CYP3A4 [21]. Additionally, PGRMC1 is able to activate CYP19 aromatase [22]. PGRMC1 is shown to physiologically affect cholesterol/steroid biosynthesis and metabolism [19]. It is known that PGRMC2 has similar interaction potential, alters activity of CYP3A4 as possible electron donor, and binds CYP21A2 [23, 24]. Most recently, both PGRMC1 and PGRMC2 were identified as putative interacting partners of ferrochelatase, an enzyme catalyzing the terminal step in the heme biosynthetic pathway, thereby possibly controlling heme release as chaperone or sensor [25]. Interaction of ALADIN with PGRMC2 at the perinuclear ER could influence CYP P450 enzyme activity through electron transfer from $\mathrm{NADPH}$ and/or control heme synthesis. In triple A syndrome, altered CYP P450 enzyme activity would consecutively contribute to adrenal atrophy [14].

Human PGRMC1 and PGRMC2 share 67\% of their protein sequence $[15,26]$. Deficiency of either PGRMC1 or PGRMC2 decreases the anti-apoptotic and antimitotic action of progesterone [27]. Additionally, increased expression of PGRMC1 or PGRMC2 inhibits entry into cell cycle $[27,28]$. On the one hand, PGRMC1 is distributed with $\beta$ - and $\gamma$-tubulin to the mitotic bipolar spindle and spindle poles in metaphase cells and on the other hand, with Aurora kinase B in meiotic cells $[29,30]$. Furthermore, PGRMC1 is thought to regulate microtubule stability [28]. PGRMC2 is shown to localize to the mitotic spindles in metaphase and anaphase cells and shall interact with cyclin-dependent kinase 11B (p58) [28]. PGRMC1 and PGRMC2 are reported to interact and furthermore, to bind to each other during metaphase, thereby suppressing entry into cell cycle [27].

Interestingly, in a large scale interactome mapping of the centrosome-cilium interface the nucleoporin ALADIN and both membrane-associated progesterone receptors PGRMC1 and PGRMC2 have been identified to localize to the cilium transition zone $[31,32]$. The centrosome is an important regulator of cell cycle progression and mitotic spindle assembly [33]. Furthermore, ALADIN is strongly dephosphorylated during mitotic exit, whereas PGRMC1 and PGRMC2 are phosphorylated during early mitotic exit [34, 35]. Obviously, ALADIN, PGRMC1 and PGRMC2 seem to have critical roles for the formation and function of the mitotic spindle apparatus in somatic cells.

Here, we report that proliferation in human adrenal cells is decreased after increased expression of ALADIN, PGRMC1 or PGRMC2. We show that PGRMC1 localizes to the microtubule kinetochore-fibers in metaphase and to the midbody in telophase of human adrenal cells and fibroblasts. Further, we present that PGRMC1 and Aurora kinase A are mislocalized in metaphase triple A patient fibroblasts. We observed that patient fibroblasts present with increased expression of PGRMC2 and we hypothesize that a depletion of ALADIN in these cells leads to a dysregulation of PGRMC2 and displaces PGRMC1 at the metaphase spindle.

\section{Results}

\section{Adrenal cell proliferation is decreased} upon over-expression of ALADIN, PGRMC1 or PGRMC2 and down-regulation of ALADIN

It is well documented that triple A patient fibroblasts show decreased cellular proliferation and doubling time [36]. To test whether depletion of ALADIN also results in decreased cellular proliferation in adrenal cells we used inducible adrenocortical NCI-H295R1TR cells stably expressing $A A A S$ shRNA (AAAS knock-down) and monitored cellular proliferation using live cell imaging for at least $65 \mathrm{~h}$. AAAS knockdown resulted in decreased proliferation in adrenal cells (growth constant $\mathrm{k}$ (slope of linear regression line $)=0.044$ ) compared to control cells expressing a scrambled shRNA $(k=0.062)$ (wild-type cells: $k=0.2)$ (Fig. 1a). Surprisingly, in live cell imaging stable overexpression of N-terminal-GFP-tagged ALADIN in adrenocortical NCI-H295R cells also impaired cellular proliferation $(\mathrm{k}=0.082)$ compared to over-expression of GFP alone in these cells $(\mathrm{k}=0.305)$ (wild-type cells: $k=0.16$ ) (Fig. 1b). Furthermore, during live cell imaging we observed that transient over-expression of C-terminal-GFP-tagged PGRMC2 in adrenocortical 


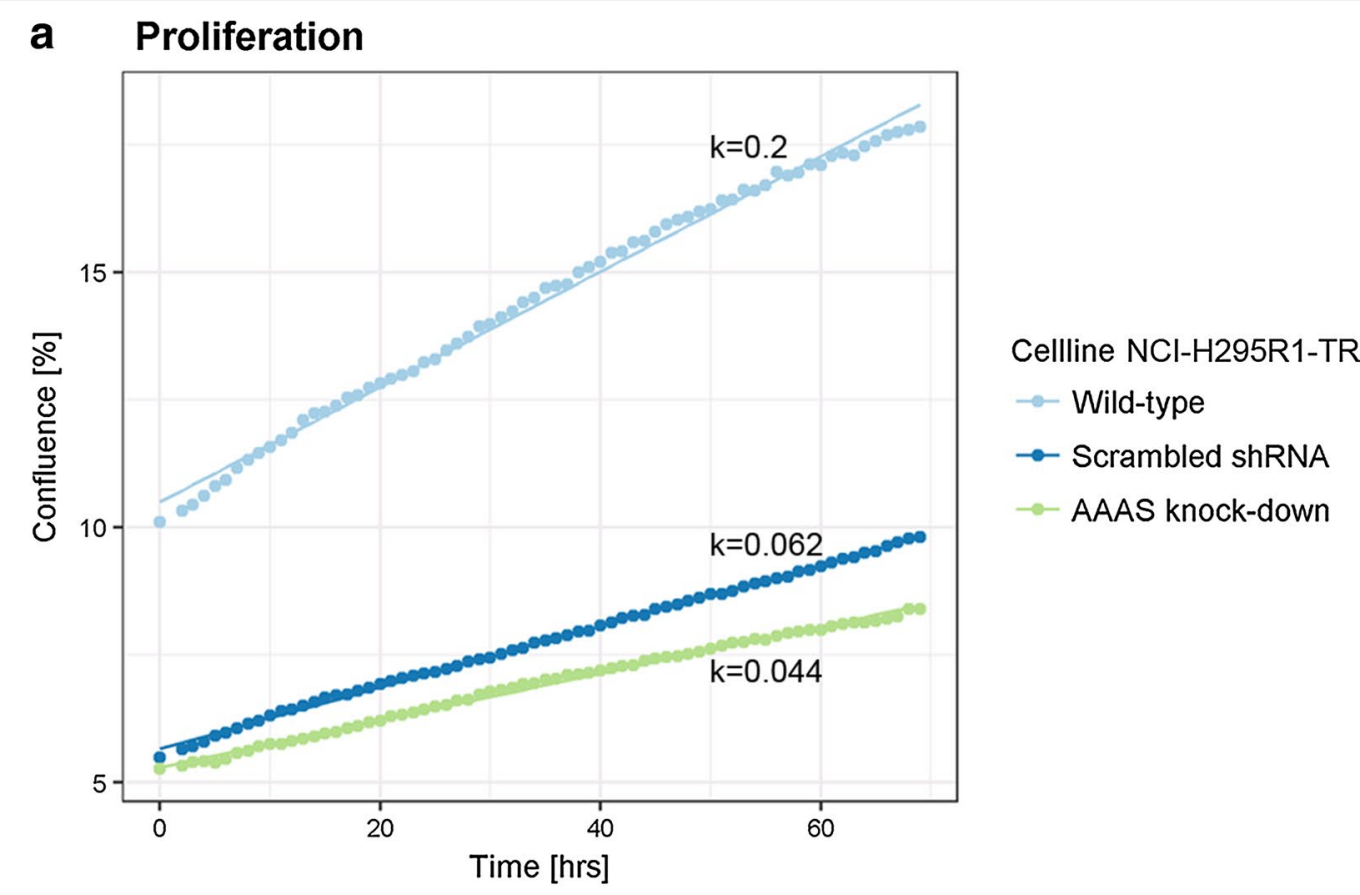

\section{b Proliferation}

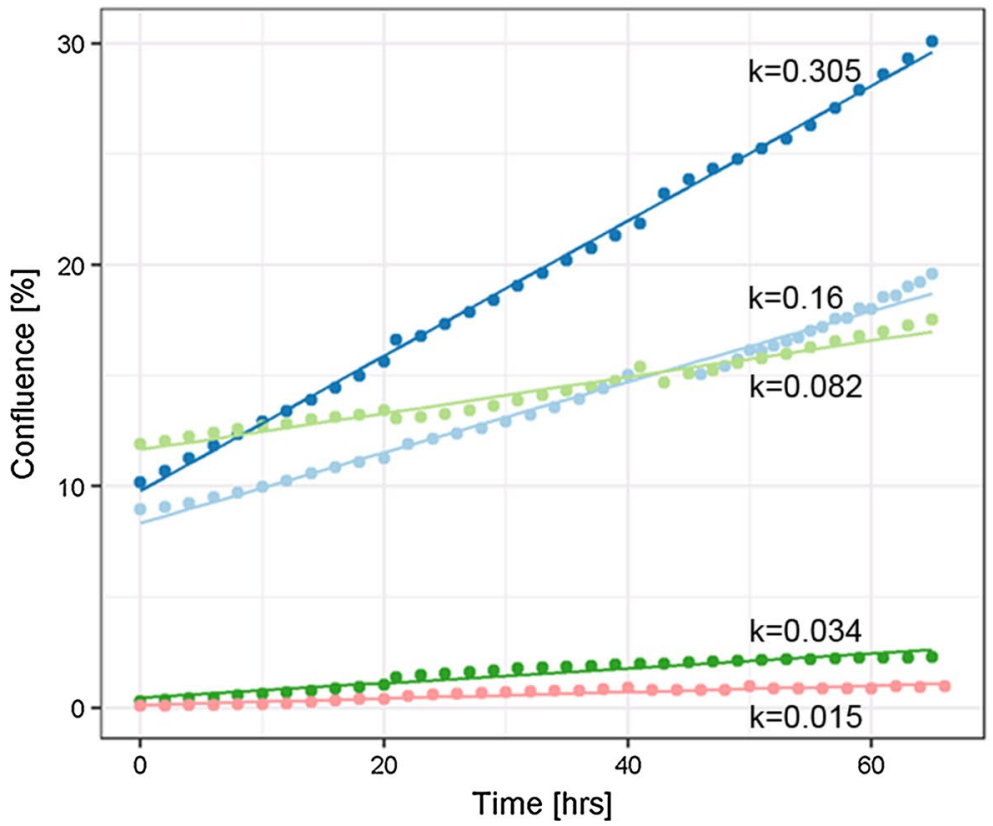

Cellline $\mathrm{NCl}-\mathrm{H} 295 \mathrm{R}$

$\rightarrow$ Wild-type

$\rightarrow$ GFP

$\because$ GFP-ALADIN

- PGRMC2-GFP

$\because$ GFP-PGRMC1

Fig. 1 Cellular proliferation is impaired upon over-expression of ALADIN, PGRMC1 or PGRMC2. Equal cell numbers of a wild-type NCI-H295R1-TR, scrambled shRNA and AAAS shRNA (AAAS knock-down) expressing NCI-H295R1-TR cells and of b wild-type NCI-H295R, GFP, GFP-ALADIN, PGRMC2-GFP and GFP-PGRMC1 expressing NCl-H295R cells were seeded $48 \mathrm{~h}$ before confluence measurement using live cell imaging on IncuCyte Zoom (Essen BioScience). In case of transient transfection with PGRMC2-GFP and GFP-PGRMC1 the proliferation of fluorescent cells which starts at cell density of about zero was measured. Growth curve analysis and growth constant k (slope of regression curve) calculation was done using multilevel regression technique using R Studio $[58,59]$ 
NCI-H295R cells resulted in decreased cellular proliferation $(k=0.034)$ (Fig. $1 \mathrm{~b})$. The same phenotype was seen in adrenocortical cells transiently over-expressing $\mathrm{N}$-terminal-GFP-tagged PGRMC1 $(\mathrm{k}=0.015)$ (Fig. 1b).

Overall, using growth curve analysis (GCA) we modeled different multilevel regression curves (Additional file 1: Figure S1, S2) and established, by comparing these models in statistical analysis, a linear model (m.1) which implies significant effects of the different cell lines (i.e. $A A A S$ knock-down) on proliferation. Thus, over-expression of ALADIN, PGRMC1 or PGRMC2 and down-regulation of ALADIN has a significant effect on adrenal cell proliferation.
PGRMC1 is restricted to metaphase spindle during mitosis and mid-body during cytokinesis

Next, we determined the localization of PGRMC1 and PGRMC2 during cell division in adrenal cells. Firstly, using immunofluorescence in adrenal NCI-H295R cells we show that PGRMC1 throughout prophase and metaphase localized to the centrosome, bipolar spindle and spindle poles (Fig. 2a). During anaphase PGRMC1 localized diffusely around chromosomes and in telophase before cytokinesis PGRMC1 localized to the mid-body (Fig. 2a). Secondly, during prophase PGRMC1 partially co-localized with mitotic Aurora kinase A (AURKA) to the centrosome and during metaphase and telophase

a
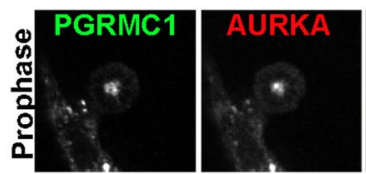

$\mathrm{NCl}-\mathrm{H} 295 \mathrm{R}$
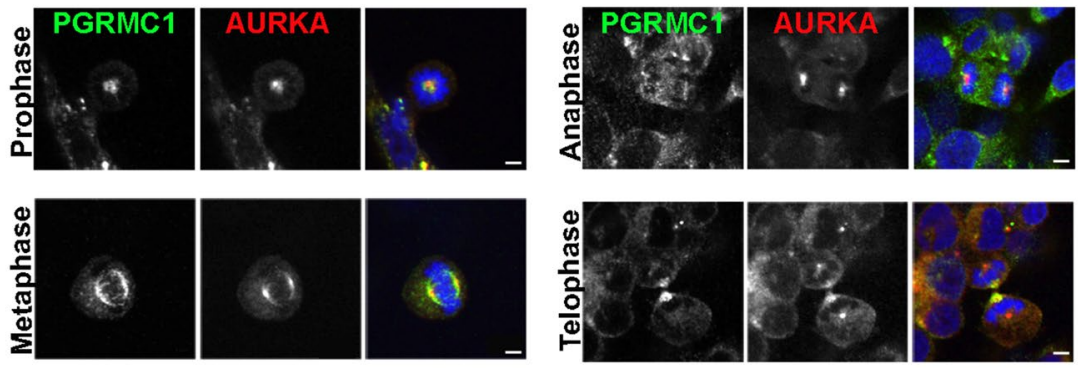

b
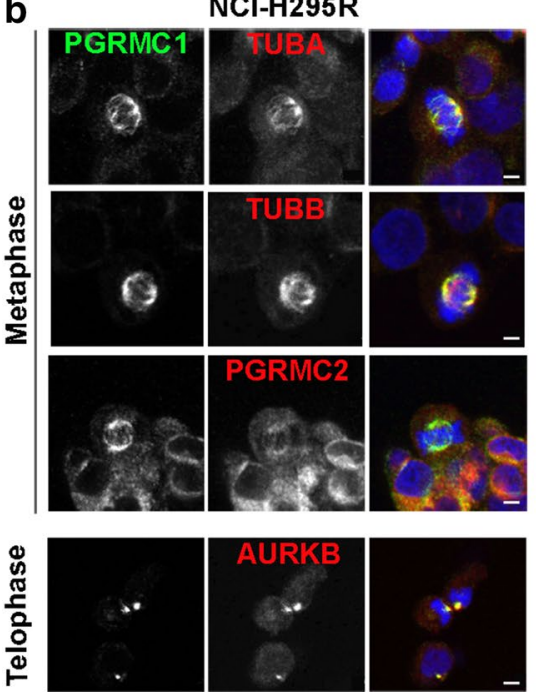

c
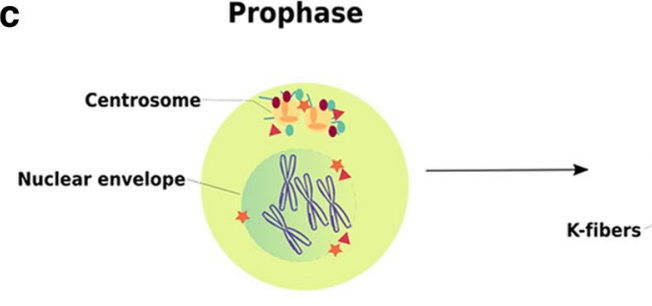

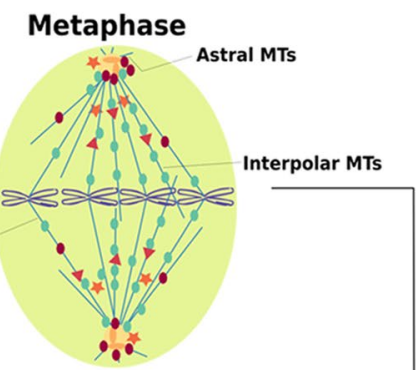

Telophase/

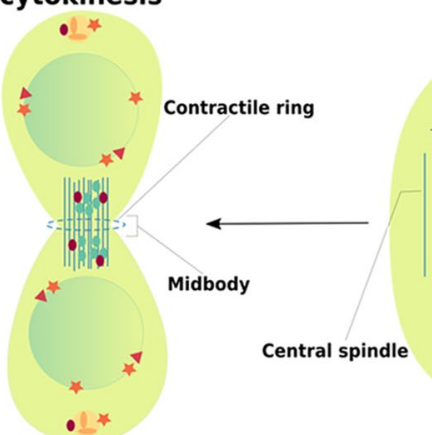
cytokinesis

Anaphase

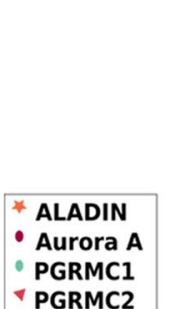

Fig. 2 PGRMC1 is restricted to the mitotic spindle and to the mid-body during cytokinesis. a Human adrenocortical NCl-H295R cells at different cell division stages were stained with anti-PGRMC1 (green), anti-Aurora kinase A (AURKA) (red) and DAPI (blue). b Human adrenocortical NCI-H295R cells at meta- and telophase were stained with anti-PGRMC1 (green), anti-a-tubulin (TUBA) (red), anti- $\beta$-tubulin (TUB) (red), anti-PGRMC2 (red), anti-Aurora kinase B (AURKB) (red) and DAPI (blue). Scale bars: $5 \mu \mathrm{m}$. c Schematic drawing of cellular localization of ALADIN, Aurora kinase A, PGRMC1 and PGRMC2 during mitosis. MT, microtubule 
to spindle poles and mid-body (Fig. 2a). During telophase and cytokinesis PGRMC1 could also be found with mitotic Aurora kinase B (AURKB) at the mid-body (Fig. 2b).

PGRMC1 efficiently localized with $\alpha$ - (TUBA) and $\beta$-tubulin (TUBB) to metaphase bipolar spindles in adrenal NCI-H295R cells (Fig. 2b). Additionally, we show that PGRMC2 localized slightly to the mitotic spindle in adrenal metaphase cells and partly co-localized with PGRMC1 during mitosis (Fig. 2b, c).

\section{Loss of ALADIN results in mis-localization of PGRMC1 and Aurora kinase $A$ during metaphase}

Since both ALADIN and PGRMC2 localized diffusely in mitotic cells compared to PGRMC1 (Fig. 2c), we assumed that ALADIN and PGRMC2 possibly exploit a regulatory role during cell division. We recently showed that a depletion of ALADIN in triple A patient fibroblasts affects the localization of PGRMC2 at the perinuclear ER [14]. Thus, we now tested the effect of over-expression or depletion of ALADIN on the localization of PGRMC1 and Aurora kinase A. We used stable GFP-ALADIN over-expressing and inducible $A A A S$ knock-down adrenocortical cells as well as skin fibroblasts from triple A patients. For this purpose, we chose patient cells with three different, and in vivo frequently occurring homozygous mutations in the $A A A S$ gene: a donor splice mutation IVS14 $+1 \mathrm{G}>\mathrm{A}$ (IVS14), a point mutation c.787T $>C$ leading to the missense mutation S263P and a point mutation c.884G > A leading to the nonsense mutation W295X.

Using immunofluorescence we revealed that stable GFP-ALADIN over-expressing adrenocortical NCIH295R cells failed to correctly localize Aurora kinase A to metaphase spindle poles (Fig. 3a, b, top panels). Aurora kinase A was not restricted to spindle poles in these cells but spread outward onto microtubule (MT) spindles compared to control cells over-expressing GFP (Fig. 3a, b, top panels). The same phenotype could be seen in inducible adrenocortical NCI-H295R1-TR cells depleted for ALADIN compared to control cells expressing scrambled shRNA (Fig. 3a, b, middle panels). Localization of PGRMC1 was additionally altered in adrenocortical cells depleted for ALADIN (Fig. 3a, b, middle panels). PGRMC1 was still correctly targeted to the bipolar MT spindle but distribution onto spindle fibers did not fully extend onto chromosomal kinetochores compared to control scrambled shRNA-expressing cells in adrenocortical cells depleted for ALADIN (Fig. 3a, b, middle panels).

Next, we tested our assumptions using fibroblasts from triple A patients. It has been described that triple A patient fibroblasts present with disorganized metaphase plates, shorter MT spindles and less active Aurora kinase A at spindle poles [7]. Here, we show in patient fibroblasts that ALADIN depletion resulted in poleward spread of Aurora kinase A onto metaphase spindles and moreover, in accumulation in the cytoplasm (Fig. 3a, b, bottom panels). The highest accumulation of Aurora kinase A in the cytoplasm was seen in patient cells carrying the ALADIN missense mutation S263P and the nonsense mutation W295X (Fig. 3a, b, bottom panels). PGRMC1 was correctly restricted to the metaphase bipolar spindle with little decreased spread in direction of kinetochore chromosomes as seen in adrenocortical cells depleted for ALADIN (Fig. 3a, b, bottom panels). Nevertheless, PGRMC1 also accumulated in the cytosol of triple A fibroblasts with highest levels in patient cells carrying the ALADIN missense mutation S263P and the nonsense mutation W295X (Fig. 3a, b, bottom panels). Thus, immunofluorescence results in triple A patient fibroblasts verified our previous findings and patient cells even presented with a more profound phenotype regarding Aurora kinase A and PGRMC1 mis-localization than adrenocortical ALADIN knock-down cells.

Our earlier work presented that depletion of ALADIN alters the localization of PGRMC2 in triple A patient fibroblasts and leads to an increased level of PGRMC2 at the perinuclear ER [14]. Furthermore, we showed that adrenal tissue of female ALADIN knock-out mice exploits higher levels of PGRMC2 protein compared to female wild-type animals [14]. Now, we show that fibroblasts from triple A patients carrying the ALADIN missense mutation S263P or the nonsense mutation W295X had about two-fold increased levels of PGRMC2 on mRNA and protein level compared to anonymized healthy control fibroblasts (Fig. 4a, b; Additional file 1: Figure S3). Cells from the patient carrying the donor splice mutation IVS14 presented also with increased protein levels of PGRMC2 but quantitative real-time PCR data was not significant (Fig. 4a, b; Additional file 1: Figure S3). Levels of PGRMC1 mRNA were not altered in these cells (Additional file 1: Figure S4).

\section{PGRMC1 localizes to microtubule kinetochore fibers}

From previous work we know that a loss of ALADIN negatively affects kinetochore (K)-fiber stability [7]. As PGRMC1 localized to the metaphase bipolar spindle, we were eager to find out whether PGRMC1 localizes to a distinct subclass of MTs during mitosis. We exposed cells before immunostaining to cold and observed whether PGRMC1 was still targeted to the spindle in mitotic cells. A positive result would exclude a localization of PGRMC1 to astral or interpolar MTs and would emphasize a role of PGRMC1 at cold stable K-fibers. Furthermore, we targeted known K-fiber proteins during immunofluorescence and observed the localization 


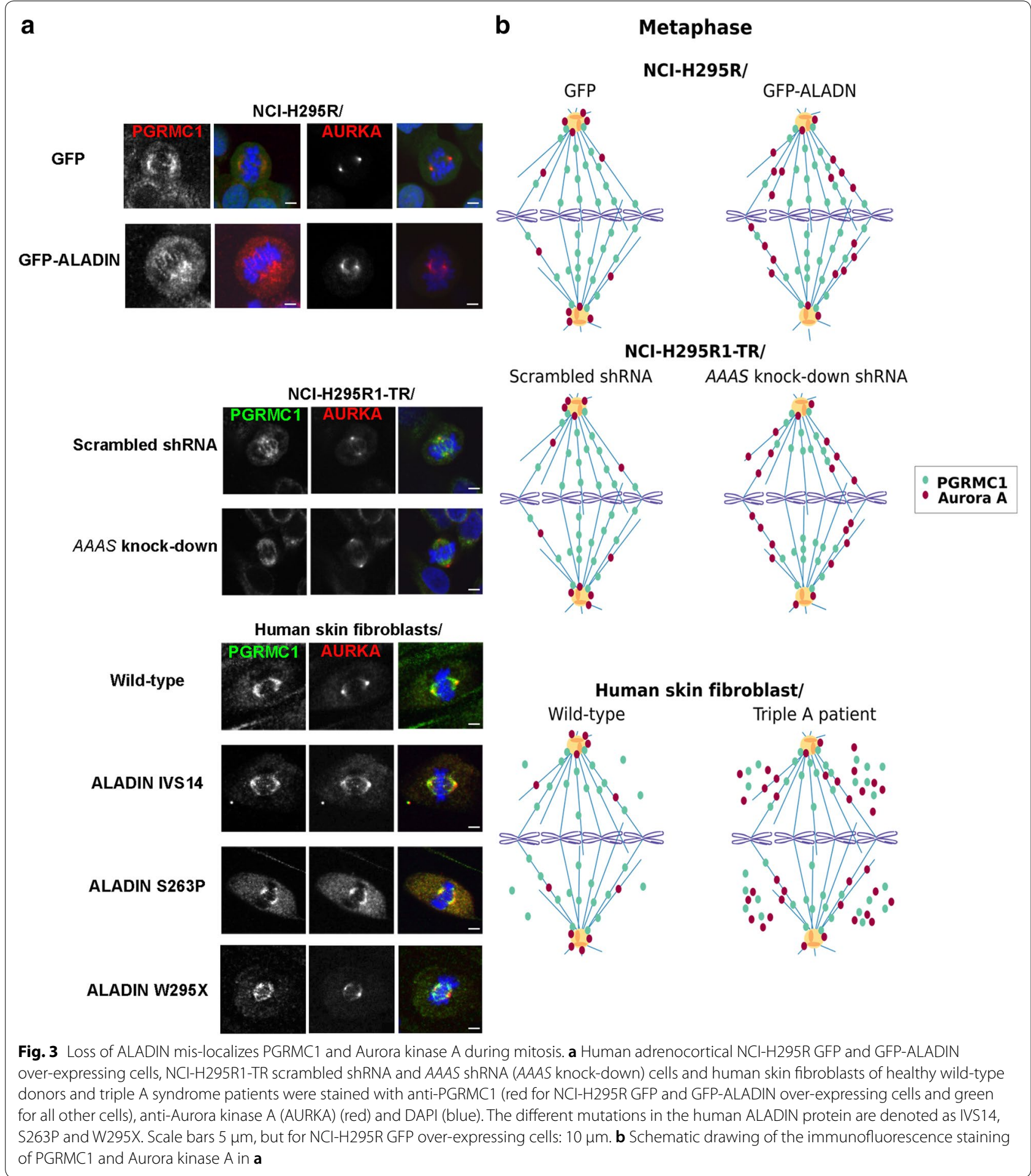

of PGRMC1 compared to these. These K-fiber proteins were first, TACC3 (also known as maskin) which is together with clathrin and chTOG (also known as CKAP5) responsible for K-fiber bundling and second,
NDC80 which belongs to the KMN complex (KNL1MIS12-NDC80 complex) that attaches K-fiber plusends to the outer chromosomal kinetochore through polymerization and de-polymerization [37, 38]. In 


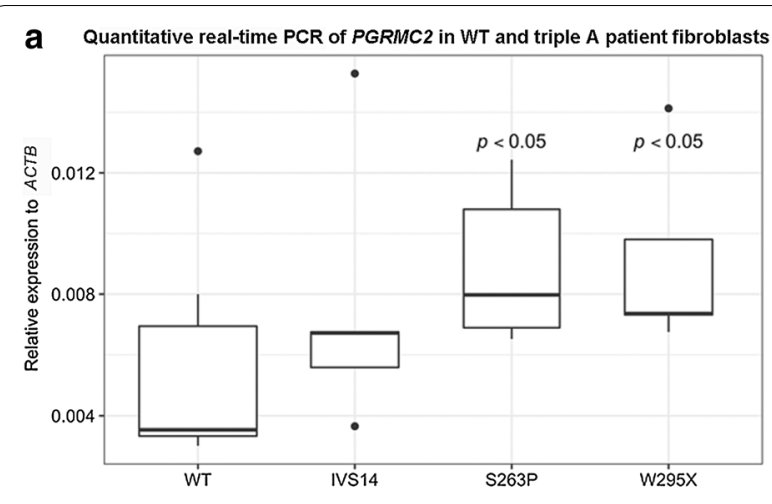

b WT $\quad$ WT $\quad$ IVS14 S263P W295x

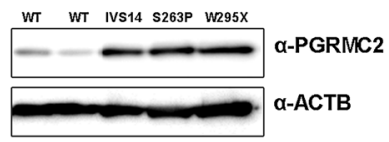

Fig. 4 PGRMC2 is over-pressed in triple A patient fibroblasts. a Total RNA was isolated from human skin fibroblasts of healthy wild-type donors and patients with triple A syndrome. The different mutations in the human ALADIN protein are denoted on the $x$-axis of the diagram: IVS14, S263P and W295X. WT, wild-type. Significant differences were measured with unpaired Wilcoxon-Mann-Whitney U-test. Boxplot widths are proportional to the square root of the samples sizes. Whiskers indicate the range outside 1.5 times the inter-quartile range (IQR) above the upper quartile and below the lower quartile. Outliers were plotted as dots. b Total protein was isolated from human skin fibroblasts of healthy wild-type donors and triple A patients followed by western blot with indicated antibodies

order to visualize the MT spindle we also stained for $\alpha$-tubulin (TUBA) and to observe the centromeric region we stained for CENPB [39]. We used adrenocortical NCI-H295R cells and additionally verified our results in a different cell type using human skin fibroblasts from healthy donors and from earlier mentioned triple A patients.

In Fig. 5a, b, we document that after cold treatment PGRMC1 was still restricted to MT spindles in adrenocortical NCI-H295R cells and human skin fibroblasts. $\alpha$-tubulin still co-localized with PGRMC1 and thus, targeted PGRMC1 to cold-stable K-fibers (Fig. 5a, b). Furthermore, immunofluorescence of CENPB marked the centromeric region of the condensed chromosomes containing the kinetochore. It can be seen that PGRMC1positive spindle K-fibers reached out to the centromeric region being attached by the KMN complex visualized by immunostaining of NDC80 (Fig. 5a, b). Immunofluorescence of PGRMC1 further partly co-localized with staining of the MT-bundling protein TACC3 in adrenocortical cells and skin fibroblasts (Fig. 5a, b). In Fig. 5c the same immunofluorescence staining pattern can be seen in cold-treated skin fibroblasts from triple A patients carrying the donor splice mutation IVS14, the missense mutation S263P or the nonsense mutation W295X. However, these cells presented with shorter and more diffuse K-fibers compared to fibroblasts from healthy donors (Fig. 5b, c).

\section{Discussion}

In primary skin fibroblasts of triple A patients the cellular proliferation rate is decreased and doubling time of patient cells is significantly increased compared to cells of healthy donors [36]. Additionally, patient cells show features of senescence, even though these cells have not reached replicative senescence as it has been documented for normal skin fibroblasts [36, 40]. The nucleoporin ALADIN is ubiquitously expressed with predominant expression in adrenal gland, gastrointestinal and central nervous system structures [41]. That may be a reason why patients with triple A syndrome present with characteristic pathogenesis in distinct tissues: adrenal insufficiency, achalasia, alacrima and involvement of the central, peripheral and autonomous nervous system [11]. In order to address the pathogenesis in adrenal tissue in the patients, we reported that loss of ALADIN leads to an impairment of glucocorticoid and mineralocorticoid synthesis in adrenal cells in vitro [42]. Here, we additionally show that ALADIN knock-down and over-expression decreases cellular proliferation in adrenal cells. Thus, it can be assumed that an equilibrated level of ALADIN protein is prerequisite for successful cellular proliferation and that depletion or accumulation of the nucleoporin impairs cellular proliferation. Carvalhal et al. already postulated that an equilibrated level of ALADIN is critical for cell division [7].

We firstly investigated, whether cellular proliferation defects are caused by diminished expression of the nucleoporin ALADIN. Secondly, we wanted to know whether an over-expression of PGRMC2, a novel discovered interactor of the nucleoporin ALADIN, also impairs cellular proliferation. In ovarian granulosa cells levels of PGRMC2 decrease during G1 phase of cell cycle [28]. Increased expression of PGRMC1 or PGRMC2 in these cells is documented to suppress entry into cell cycle [27, 28]. During metaphase both proteins are thought to interact and to impair progression of cell cycle [27]. We show here that over-expression of PGRMC1 or PGRMC2 also lead to decreased proliferation in adrenal cells. These results are in accordance with the literature and emphasize a simultaneous role of PGRMC1 and PGRMC2 during cellular proliferation. Mitotic errors in these cells could lead to apoptosis but future research shall address the exact reason for the decreased cellular proliferation upon over-expression of PGRMC1 and/or PGRMC2. 


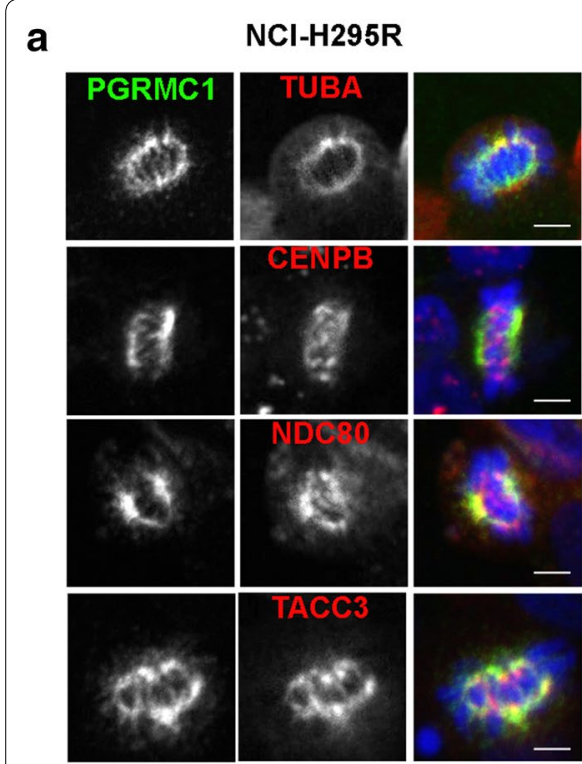

C

\section{Human skin fibroblasts/} ALADIN IVS14
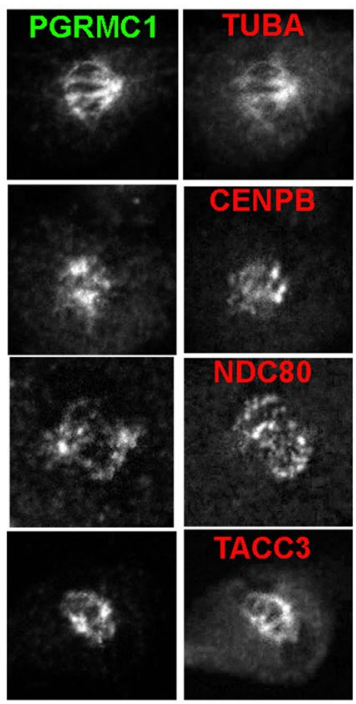

b

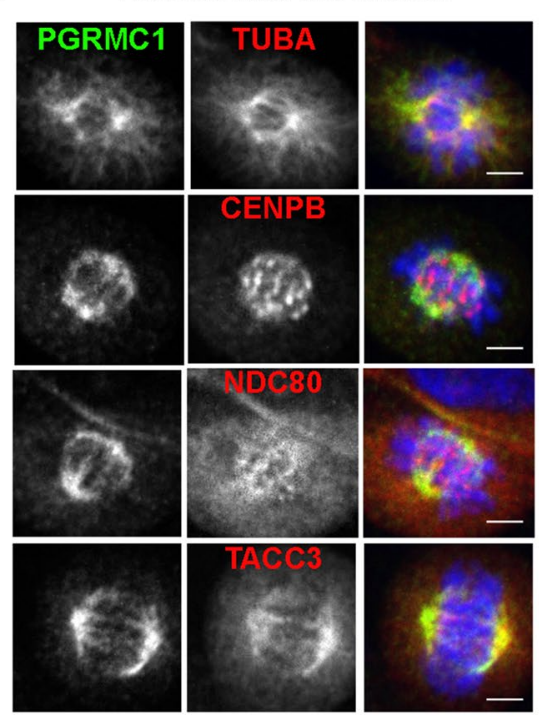

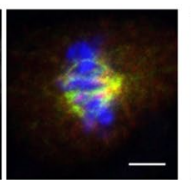

Human skin fibroblasts/ ALADIN S263P
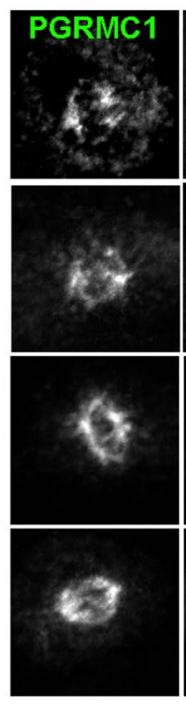
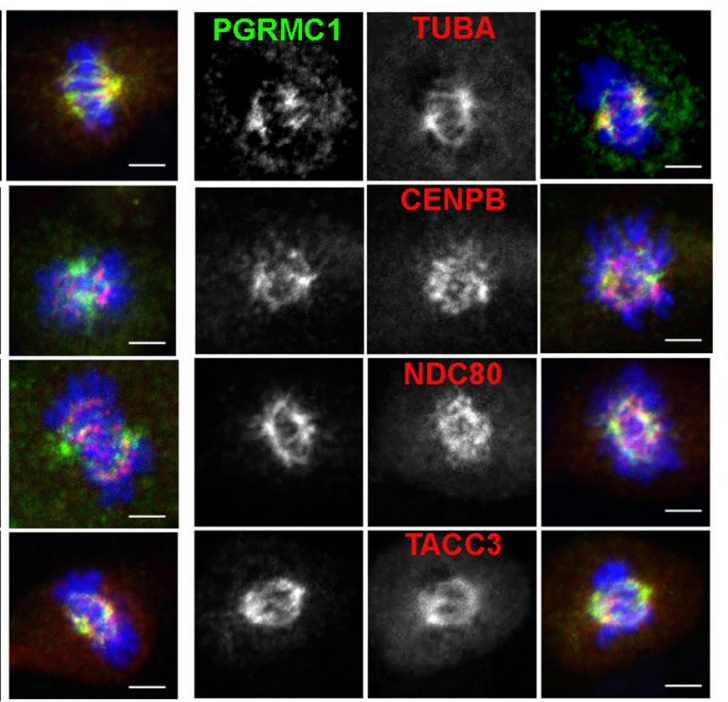

Human skin fibroblasts/ ALADIN W295X

Fig. 5 PGRMC1 localizes to cold-stable kinetochore fibers. Cells were cold-treated and stained with anti-PGRMC1 (green), anti-a-tubulin (TUBA) (red), anti-CENPB (red), anti-NDC80 (red), anti-TACC3 (red) and DAPI (blue). a Human adrenocortical NCl-H295R cells. b Human skin fibroblasts of healthy wild-type donors. c Human skin fibroblasts of triple A patients. The different mutations in the human ALADIN protein are denoted as IVS14, S263P and W295X. Scale bars $5 \mu \mathrm{m}$

We present that over-expression of ALADIN, PGRMC1 or PGRMC2 leads to an impairment of adrenal cellular proliferation. Thus, we assumed that each of these proteins plays an important role during regulation of cell division. It is known that ALADIN is a novel regulator of mitotic controller Aurora kinase A [7]. Humans have three classes of serine/threonine Aurora kinases: Aurora
A, Aurora B and Aurora C [43]. The homologous Aurora kinase A and Aurora kinase B are expressed in most cell types. Despite different localization and activation during cell cycle, they both regulate progression through cell cycle from G2 phase to cytokinesis [43]. In Drosophila ALADIN localizes diffusely through the spindle during mitosis [7]. In HeLa cells ALADIN could be found within 
the mitotic spindle accumulated at spindle poles but the highest amount appeared between centrosome and metaphase plate (schematically summarized in Fig. 2c) [7]. This high concentration of ALADIN protein is thought to be an ER-membrane-associated pool of the nucleoporin since it partially co-localizes with calnexin which is an integral microsomal protein [7]. PGRMC1 and PGRMC2 are documented to localize to the mitotic spindle apparatus in metaphase ovarian granulosa cells but their role at the spindle apparatus and during cell cycle is not known [28, 30]. PGRMC1 has been documented to associate with Aurora kinase B during meiosis but no such role has been assigned during mitosis [29]. Both Aurora kinase A and Aurora kinase B phosphorylate and thereby regulate a variety of mitotic spindle substrates. Dysregulation of these kinases results in fatal mitotic errors [43]. Aurora kinase A has distinct roles in centrosome maturation, entry into mitosis, spindle assembly and microtubule (MT) function during anaphase. Aurora kinase B is essential for bi-orientation of chromosomes and cytokinesis [44]. In our results we show that PGRMC1 localizes to the MT kinetochore (K)-fibers in metaphase and to the midbody in telophase of human adrenal cells and fibroblasts. Furthermore, we find that PGRMC1 co-localizes with Aurora kinase A and Aurora kinase B. It is tempting to assume that PGRMC1 is a substrate being phosphorylated by one or both of these mitotic kinases and indeed, PGRMC1 has been found to be phosphorylated at three postulated serines during early mitotic exit [35]. Further research should address whether PGRMC1 is phosphorylated by Aurora kinases and should uncover the regulatory effect during mitosis of phosphorylation on one of the three postulated serines in PGRMC1.

It has already been documented that PGRMC1 interacts with $\beta$-tubulin that is one of the two main components of MTs which are built from heterodimers of $\alpha$ - and $\beta$-tubulin $[30,33]$. Heterodimers are arranged in a head-to-tail fashion into protofilaments whereby in humans 13 of such protofilaments laterally arrange into tubular structures of about $25 \mathrm{~nm}$ diameter [45]. Within MTs $\alpha$ - and $\beta$-tubulin heterodimers reveal a distinct intrinsic polarity in which the minus-end confers to the end where $\alpha$-tubulin is exposed and the plus-end where $\beta$-tubulin is exposed [45]. Microtubule polymerization is done at the $\beta$-tubulin plus-end and initial nucleation of MTs is facilitated by $\gamma$-tubulin including a complex of several proteins [45]. Microtubules undergo dynamic cycles of polymerization and de-polymerization which is called dynamic instability and is achieved by a variety of regulatory proteins [45]. Co-localization of PGRMC1 with $\beta$ - and $\gamma$-tubulin has been proposed before, but a direct interaction of PGRMC1 could only be shown for $\beta$-tubulin [30]. Here, we show that $\beta$-tubulin is a positive target for a novel interaction with PGRMC1 and that association of PGRMC1 with $\beta$-tubulin possibly influences polymerization at plus-ends of MTs during mitosis. In interphase cells we previously reported that PGRMC2 co-localizes with the nucleoporin ALADIN to the perinuclear space [14]. Here, it appeared that PGRMC2 like ALADIN localizes diffusely in mitotic cells and that only PGRMC1 could be efficiently restricted to the mitotic bipolar spindle (schematically summarized in Fig. 2c). It is known that PGRMC1 and PGRMC2 interact to suppress entry into cell cycle [27]. With immunofluorescence we could however not target PGRMC2 to the same site as PGRMC1 during mitosis. Thus, we hypothesize that PGRMC2 plays a distinct role in regulating the function of PGRMC1 at the mitotic spindle and presumably during cell division.

ALADIN is a new important co-factor of mitotic controller Aurora kinase A [7]. Aurora kinase A normally resides at the centrosome and spindle poles where it regulates centrosome maturation and MT spindle assembly [44]. Carvalhal et al. reported that mitotic HeLa cells depleted for or over-expressing ALADIN have less Aurora kinase A at centrosomes and higher amounts of it at spindle fibers [7]. We present that ALADIN depletion and over-expression in adrenocortical cells and in triple A patient fibroblasts results in mislocalization of Aurora A to MT spindles. Our findings further prove that ALADIN partially regulates mitotic Aurora kinase A and raise the question why an equilibrated level of ALADIN protein is of great importance for proper localization of Aurora kinase A. We have earlier shown that ALADIN interacts with PGRMC2 and that triple A patients have increased levels of PGRMC2 at the perinuclear ER [14]. It is thought that PGRMC2 binds its protein homologue PGRMC1 resulting in suppression of cell cycle [27]. Our results show that only depletion of ALADIN and not over-expression affected localization of PGRMC1 in mitotic cells. We know that ALADIN directly regulates Aurora kinase A and therefore, any change in level of ALADIN protein alters Aurora kinase A localization and function during mitosis. As only depletion of ALADIN alters localization of PGRMC1 in immunofluorescence, it can be assumed that during mitosis ALADIN not directly regulates PGRMC1 but does so rather through secondary mechanisms.

We hypothesize that increased levels of PGRMC2 in fibroblasts from triple A patients and in ALADIN KO mice result from the loss of ALADIN [14]. Furthermore, we assume since ALADIN is a novel interactor of PGRMC2 that ALADIN negatively regulates PGRMC2. A direct or indirect negative regulation of PGRMC2 results in accumulation of PGRMC2 upon ALADIN depletion. We revealed that ALADIN depleted patient 
fibroblasts fail to correctly target PGRMC1 fully to the mitotic spindle fibers, instead PGRMC1 accumulates in the cytosol. We already presented that over-expression of either PGRMC1 or PGRMC2 impairs cellular proliferation. Based on the finding that PGRMC1 interacts with PGRMC2, we think that increased levels of PGRMC2 displace PGRMC1 from its correct localization at the mitotic spindle and target PGRMC1 with PGRMC2 to the cytosol impairing cell division and proliferation. Over-expression of PGRMC1 alone would probably also result in the same phenotype while targeting high amount of PGRMC1 to the cytoplasm.

Adrenocortical ALADIN knock-down cells did not present with alteration of PGRMC2 expression [14]. Additionally, in immunofluorescence during mitosis PGRMC1 and Aurora kinase A were not targeted to the cytosol in these cells (Fig. 3a, b, middle panels). Moreover, fibroblasts from the patient carrying the donor splice mutation IVS14 had less PGRMC1 and Aurora kinase A targeted to the cytosol (Fig. 3a, b, bottom panels). Future research has to address in more detail whether the loss of regulation of PGRMC2 and Aurora kinase A is dependent on different levels in ALADIN protein and on different kinds of mutations in the $A A A S$ gene.

The mature bipolar mitotic spindle is made from different subclasses of MTs depending on their position, functionality and organization: astral MTs, interpolar MTs and K-fibers (Fig. 1c) [45]. Astral MTs connect the centrosome with the cell cortex and regulate centrosome separation and spindle positioning [46]. However, it has been shown that mitosis can occur without astral MTs $[47,48]$. The main body of the mitotic spindle is made up by dynamic interpolar MTs. Interpolar MTs originate from the centrosome towards the center of the spindle and connect in an anti-parallel fashion with interpolar MTs originating from the opposite spindle pole [45]. Some interpolar MTs however are shorter and do not emanate have way through the mitotic spindle [49]. Interpolar MTs are the main component of the central spindle during anaphase and maintain spindle polarity and chromosome segregation [50,51]. However, interpolar MTs only indirectly establish chromosome segregation because this function is facilitated by $\mathrm{K}$-fibers [45]. K-fibers are big bundles of 20-40 parallel MTs and are responsible of chromosomes attachment to spindle poles and facilitate sister chromatid segregation $[45,52]$. K-fibers are less dynamic and therefore have an average half-life of 4-8 min comparable to that of interphase MTs with 9-10 min $[45,53]$. The average half-lifes of astral and interpolar MTs are less than $1 \mathrm{~min}[45,54]$. Therefore, K-fibers are the most stable MTs when exposed to cold or depolymerizing agents like nocodazole.
Lodde and Peluso showed that PGRMC1 affects MT stability and mitotic progression by the action of progesterone [30]. We present here that PGRMC1 efficiently localizes to K-fibers upon cold treatment before immunostaining and that $\mathrm{K}$-fibers are shorter and more diffuse in fibroblasts of triple A patients. We hypothesize that the previously documented decreased stability of K-fibers upon ALADIN depletion [7] is partly the result of a mis-regulated PGRMC2 which displaces PGRMC1 from K-fibers to the cytosol.

\section{Conclusion}

With our work we have given evidence, that depletion of ALADIN impairs mitotic cell division and we can explain parts of the pathogenesis of triple A syndrome. Our new results shall be the basis for more extended research focusing on mis-regulated PGRMC2 and Aurora kinase A due to loss of ALADIN.

We document here that PGRMC1, the protein homologue of PGRMC2, localizes to cold-stable MT K-fibers in human adrenocortical cells and skin fibroblasts. We verify that K-fibers are less stable upon ALADIN loss of function in triple A patient fibroblasts and we present that these cells fail to target PGRMC1 efficiently to the mitotic spindle. Thus, we assume that part of the phenotype seen in triple A patient cells can be caused by a misregulated association between ALADIN and PGRMC2 resulting in mis-localization of PGRMC1 during mitosis and less stable K-fibers. Further studies are required to address the exact function of PGRMC1 at K-fibers and to solve the question whether it is involved in MT bundling and/or MT maturation.

\section{Methods}

\section{Cell culture}

All adrenal carcinoma NCI-H295R cells were cultured in DMEM/F12 medium (Lonza, Cologne, Germany) supplemented with $1 \mathrm{mM}$ L-glutamine (Lonza, Cologne, Germany), 5\% Nu-serum (BD Biosciences, Heidelberg, Germany), 1\% insulin-transferrin-selenium) (Gibco, Life Technologies, Darmstadt, Germany) and 1\% antibioticantimycotic solution (PAA, GE Healthcare GmbH, Little Chalfont, United Kingdom). NCI-H295R cells stably expressing GFP-ALADIN fusion protein or GFP were generated as described previously using the gamma-retroviral transfer vectors pcz-CFG5.1-GFP- $A A A S$ and pczCFG5.1-GFP [1]. NCI-H295R1-TR cells stably expressing $A A A S$ shRNA ( $A A A S$ knock-down) or scrambled shRNA were generated by our group as previously reported [42]. These cells were cultured with $100 \mu \mathrm{g} / \mathrm{ml}$ zeocin (InvivoGen, Toulouse, France) supplemented in culture medium. Doxycycline hydrochloride (MP Biomedicals, 
Eschwege, Germany) was used at $1 \mu \mathrm{g} / \mathrm{ml}$ for $48 \mathrm{~h}$ to turn on the expression of the shRNA sequence. Expression levels of ALADIN in the two adrenal cell lines were assessed previous to all our experiments; especially after de-freezing of the cells. This data is not shown due to the straight-forward characterization of the cell lines [14, 42].

Triple A patient skin fibroblasts and human anonymized control skin fibroblasts were obtained and cultured as described earlier [36]. All fibroblasts were cultured until passage 20 at the most. Informed consent was obtained from all subjects and experiments were approved by the local ethics review board (Medical Faculty, Technische Universität Dresden, EK820897).

\section{Transient adrenal cell transfection}

Cells were cultured for proliferation analysis in 24-well culture dishes at a density of $0.4 \times 10^{5}$ cells/well or for immunofluorescence microscopy onto cover slips (Carl Zeiss, Jena, Germany) in 6-well culture dishes at a density of $1.6 \times 10^{5}$ cells/well $24 \mathrm{~h}$ before subsequent transfections. Cells were transfected using X-treme GENE HP DNA transfection reagent (Roche Diagnostics, Mannheim, Germany). The plasmids for transient transfections pEGFP-C1-PGRMC1 and pCMV6-ACPGRMC2-GFP vector (RG204682) (OriGene Technologies, Rockville MD, USA) were used at a concentration of $0.01 \mu \mathrm{g} / \mu \mathrm{l}$ at an optimized transfection ratio of $1: 4$ diluted in pure DMEM/F12. Proliferation was monitored after $24 \mathrm{~h}$ after transfection. Cells for immunofluorescence were fixed after $48 \mathrm{~h}$.

\section{Proliferation analysis}

Cells were seeded in 24-well culture dishes at a density of $0.4 \times 10^{5}$ cells/well $24 \mathrm{~h}$ before proliferation analysis. Confluence measurement was done using live cell imaging on IncuCyte Zoom (Essen BioScience, Ann Arbor MI, USA) over at least $65 \mathrm{~h}$. Measurement was done at least in triplicate and experiments were repeated at least twice.

Live proliferation data was collected by the instrument and automatically averaged. GCA and growth constant $\mathrm{k}$ (slope of linear regression line) calculation was done using multilevel regression technique using $\mathrm{R}$ Studio as described earlier $[58,59]$. By using GCA we were able to analyze both group-level and individual-level effects on cellular proliferation. The different model fits have been included in the additional material: the intercept model (m.0; Additional file 1: Figure S1A, S2A) and the linear model (m.1; Additional file 1: Figure S1B, S2B). The intercept model suggests constant differences in proliferation randomly assigned to the different cell lines and the linear model suggests effects of the different cell lines on proliferation. Statistical comparison [Pearson's Chi squared test with: degrees of freedom $=2$ (Fig. 1a) and
4 (Fig. 1b)] of these two models revealed that the linear model (m.1) is our regression model of choice $(\mathrm{p}<0.001)$. A third model for regression calculation was evaluated which included unmeasured individual properties affecting cell growth but this model did not improve the regression calculation (data not shown).

\section{Immunofluorescence microscopy}

Cells grown on glass cover slips were fixed with $4 \%$ PFA in PBS for 5 min, permeabilized with $0.5 \%$ Triton$\mathrm{X}-100$ in PBS for $5 \mathrm{~min}$ and then fixed again. In order to assess K-fiber microtubule stability, cells were put on ice for $10 \mathrm{~min}$ prior fixation and permeabilization as reported elsewhere [7]. Blocking was performed with $2 \%$ BSA/0.1\% Triton-X-100 in PBS for 30 min at room-temperature (RT).

All antibodies used for immunofluorescence were diluted in blocking solution. Primary antibodies antiALADIN (B-11: sc-374073) (1:25), anti-AURKA (C-1: sc-398814) (1:50), anti-AURKB (A-3: sc-393357) (1:50), anti-CENPB (F-4: sc-376283) (1:50), anti-NDC80 (C-11: sc-515550) (1:50), anti-PGRMC1 (C-4: sc-393015) (1:50), anti-PGRMC2 (F-3: sc-374624) (1:50), anti-TACC3 (C-2: sc-376883) (1:50), anti-TUBA (DM1A: sc-32293) (1:50) and anti-TUBB (D-10: sc-5274) (1:50) (Santa Cruz Biotechnology Inc., Heidelberg, Germany) were incubated at $4{ }^{\circ} \mathrm{C}$ over-night in a humidified chamber. Secondary antibodies Alexa Fluor 488 goat anti-mouse IgM and Alexa Fluor 568 goat anti-mouse IgG (1:500) (Molecular Probes, Life Technologies) were incubated $1 \mathrm{~h}$ at RT in the dark. Excess antibodies after primary and secondary antibody staining were removed by three washing steps using $0.1 \%$ Triton-X-100 in PBS for 5 min. Cover slips were mounted onto microscope slides with VECTASHIELD mounting medium for fluorescence with DAPI (Vector Laboratories, Burlingame, CA, USA). Negative controls (secondary fluorescent antibody only) were conducted for background assessment and LASER power regulation (data not shown).

Fluorescence was imaged using the confocal laser scanning microscope Zeiss LSM 510 with Zeiss EC PlanNeofluar $40 \times$ objective/1.3 Oil and the following lasers: diode $405 \mathrm{~nm}$, Argon $488 \mathrm{~nm}$ and DPSS $561 \mathrm{~nm}$ (Carl Zeiss). Images were acquired and processed using equipment of the Core Facility Cellular Imaging at the Medical Theoretical Centre in Dresden. The experiments were repeated at least three times. For the adrenal cell lines approximately 50 mitotic cells were involved in the analysis, for the fibroblasts the number of analyzed cells was less (approximately 20 cells), since fibroblasts have less doubling time than cancer cells and, especially mitotic patient fibroblast divide even less [36]. 


\section{RNA extraction, CDNA synthesis and quantitative real-time} PCR

Total RNA from cultured cells was isolated using the NucleoSpin RNA (Macherey-Nagel, Düren, Germany) according to the protocol from the manufacturer. Purity of the RNA was assessed using Nanodrop Spectrophotometer (ND-1000) (NanoDrop Technologies, Wilmington DE, USA). The amount of 500 ng of total RNA was reverse transcribed using the GoScript Reverse Transcription System (Promega, Mannheim, Germany) following the protocols from the manufacturer. Primers for the amplification of the target sequence were designed using Primer Express 3.0 (Applied Biosystems, Life Technologies) and compared to the human genome database for unique binding using BLAST search [55]. Primers for $P G R M C 1$ (forward, reverse and probe) were used as previously described [56]. The primer sequences are listed in the additional data of this article (Additional file 1: Table S1).

The qPCR amplifications were performed in triplicates using the GoTaq Probe qPCR Master Mix (Promega) according to the manufacturer's reaction parameter on an ABI 7300 Fast Real-Time PCR System (Applied Biosystems, Life Technologies). In all results repeatability was assessed by standard deviation of triplicate $C_{t} s$ and reproducibility was verified by normalizing all real-time RT-PCR experiments by the $C_{t}$ of each positive control per run. The experiments were repeated at least five times.

\section{Immunoblots}

After SDS-PAGE separation onto 4-12\% PAGE (150 V for $1.5 \mathrm{~h}$ ) and electroblotting ( $30 \mathrm{~V}$ for $1.5 \mathrm{~h}$ ) (Invitrogen, Life Technologies) onto Amersham hybond-ECL nitrocellulose membrane $(0.45 \mu \mathrm{m})$ (GE Healthcare $\mathrm{GmbH}$, Little Chalfont, United Kingdom) non-specific binding of proteins to the membrane was blocked by incubation in PBS containing $3 \% \mathrm{BSA}$ at RT.

The membrane was then probed with primary antibodies either anti-ACTB (clone AC-74) (1:40,000 in 3\% PBS/BSA) (Sigma-Aldrich, Munich, Germany), antiPGRMC1 (C-4: sc-393015) (1:100 in 3\% PBS/BSA) or anti-PGRMC2 (F-3: sc-374624) (Santa Cruz Biotechnology, Inc.) (1:100 in $3 \% \mathrm{PBS} / \mathrm{BSA})$ over-night at $4{ }^{\circ} \mathrm{C}$. Secondary antibodies goat anti-mouse IgG conjugated to horseradish peroxidase (1:5000 in 3\% PBS/BSA) (Cell Signalling Technology Europe B.V., Leiden, Netherlands) were incubated $1 \mathrm{~h}$ at RT. Protein bands were detected using ECL system and visualized on autoradiography film (Hyperfilm ECL; GE Healthcare, Munich, Germany). Negative controls for the western blot were done with the secondary antibody only, when the antibody was initially tested (data not shown).

\section{Statistics}

Statistical analyses were made using the open-source software $\mathrm{R}$ version 3.4.2 and $\mathrm{R}$ Studio version 1.0.136 [57]. Unpaired Wilcoxon-Mann-Whitney U-test was performed. During evaluation of the results a confidence interval alpha of $95 \%$ and $p$ values lower than 0.05 were considered as statistically significant. Results are shown as box plots which give a fast and efficient overview about median, first and third quartile (25th and 75th percentile, respectively), interquartile range (IQR), minimal and maximal values and outliers.

\section{Additional file}

Additional file 1. Figure S1: Multi-level regression models of Figure 1A. Figure S2: Multi-level regression models of Figure 1B. Figure S3: Full-length western blot of Figure 4B. Figure S4: TaqMan analysis of PGRMC1 in human skin fibroblasts. Table S1: Real-time qPCR primer oligonucleotides.

\section{Authors' contributions}

Conceptualization: RJ, AH, KK; methodology: RJ; formal analysis: RJ, DL; investigation: RJ, DL; writing —original draft: RJ; writing—review and editing: $\mathrm{RJ}, \mathrm{AH}$, KK; supervision: $\mathrm{AH}, \mathrm{KK}$; project administration: $\mathrm{AH}, \mathrm{KK}$. All authors read and approved the final manuscript.

\section{Acknowledgements}

We thank Waldemar Kanczkowski (Technische Universität Dresden, Dresden, Germany) for providing the NCI-H295R cells. Barbara Kind (Technische Universität Dresden, Dresden, Germany) generously generated pseudo retroviruses containing pcz-CFG5.1-GFP-ALADIN and pcz-CFG5.1-GFP. Alexandra Wendler (Universität Heidelberg, Mannheim, Germany) kindly provided pEGFP-C1PGRMC1 plasmid.

\section{Competing interests}

The authors declare that they have no competing interests.

Availability of data and materials

All data and material in this manuscript are available upon request.

Consent for publication

All authors have read and agreed to the final version of the manuscript.

\section{Ethics approval and consent to participate}

For skin fibroblasts informed consent was obtained from all subjects and experiments were approved by the local ethics review board (Medical Faculty, Technische Universität Dresden, EK820897).

\section{Funding}

This work was funded by the Deutsche Forschungsgemeinschaft to $\mathrm{AH}$ : within the Clinical Research Unit 252 (project HU 895/5-2) and within the CRC/Transregio 205/1 "The adrenal: Central relay in Health and Disease". The funders had no role in study design, data collection and analysis, decision to publish, or preparation of the manuscript. We acknowledge support by the Open Access Publication Funds of the SLUB/TU Dresden.

\section{Publisher's Note}

Springer Nature remains neutral with regard to jurisdictional claims in published maps and institutional affiliations. 
Received: 18 August 2018 Accepted: 25 October 2018

Published online: 10 November 2018

\section{References}

1. Kind B, Koehler K, Lorenz M, Huebner A. The nuclear pore complex protein ALADIN is anchored via NDC1 but not via POM121 and GP210 in the nuclear envelope. Biochem Biophys Res Commun. 2009;390:205-10.

2. Yamazumi Y, Kamiya A, Nishida A, Nishihara A, lemura S, Natsume T, et al. The transmembrane nucleoporin NDC1 is required for targeting of ALADIN to nuclear pore complexes. Biochem Biophys Res Commun. 2009;389:100-4.

3. Rabut G, Doye V, Ellenberg J. Mapping the dynamic organization of the nuclear pore complex inside single living cells. Nat Cell Biol. 2004:6:1114-21.

4. Fahrenkrog B. Nucleoporin gene fusions and hematopoietic malignancies. New J Sci. 2014:2014 Journal Article: Article ID 468306.

5. Nofrini V, Di Giacomo D, Mecucci C. Nucleoporin genes in human diseases. Eur J Hum Genet. 2016:24:1388-95.

6. Sakuma S, D'Angelo MA. The roles of the nuclear pore complex in cellular dysfunction, aging and disease. Semin Cell Dev Biol. 2017;68:72-84

7. Carvalhal S, Ribeiro SA, Arocena M, Kasciukovic T, Temme A, Koehler K, et al. The nucleoporin ALADIN regulates Aurora A localization to ensure robust mitotic spindle formation. Mol Biol Cell. 2015;26:3424-38.

8. Carvalhal S, Stevense M, Koehler K, Naumann R, Huebner A, Jessberger R, et al. ALADIN is required for the production of fertile mouse oocytes. Mol Biol Cell. 2017;28:2470-8.

9. Tullio-Pelet A, Salomon R, Hadj-Rabia S, Mugnier C, de Laet MH, Chaouachi $B$, et al. Mutant WD-repeat protein in triple-A syndrome. Nat Genet. 2000:26:332-5.

10. Handschug K, Sperling S, Yoon SJ, Hennig S, Clark AJ, Huebner A. Triple A syndrome is caused by mutations in AAAS, a new WD-repeat protein gene. Hum Mol Genet. 2001;10:283-90.

11. Allgrove J, Clayden GS, Grant DB, Macaulay JC. Familial glucocorticoid deficiency with achalasia of the cardia and deficient tear production. Lancet. 1978;1:1284-6.

12. Cronshaw JM, Matunis MJ. The nuclear pore complex protein ALADIN is mislocalized in triple A syndrome. Proc Natl Acad Sci USA. 2003;100:5823-7.

13. Krumbholz M, Koehler K, Huebner A. Cellular localization of 17 natural mutant variants of ALADIN protein in triple A syndrome-shedding light on an unexpected splice mutation. Biochem Cell Biol. 2006;84:243-9.

14. Jühlen $\mathrm{R}$, Landgraf $\mathrm{D}$, Huebner $\mathrm{A}$, Koehler K. Identification of a nove putative interaction partner of the nucleoporin ALADIN. Biol Open. 2016:5:1697-705

15. Cahill MA, Medlock AE. Thoughts on interactions between PGRMC1 and diverse attested and potential hydrophobic ligands. J Steroid Biochem Mol Biol. 2017;171:11-33.

16. Falkenstein E, Meyer C, Eisen C, Scriba PC, Wehling M. Full-length CDNA sequence of a progesterone membrane-binding protein from porcine vascular smooth muscle cells. Biochem Biophys Res Commun. 1996;229:86-9.

17. Clark NC, Friel AM, Pru CA, Zhang L, Shioda T, Rueda BR, et al. Progesterone receptor membrane component 1 promotes survival of human breast cancer cells and the growth of xenograft tumors. Cancer Biol Ther. 2016;17:262-71

18. Kabe Y, Nakane T, Koike I, Yamamoto T, Sugiura Y, Harada E, et al. Haemdependent dimerization of PGRMC1/Sigma-2 receptor facilitates cancer proliferation and chemoresistance. Nat Commun. 2016;7:11030.

19. Ryu CS, Klein K, Zanger UM. Membrane Associated progesterone receptors: promiscuous proteins with pleiotropic functions-focus on interactions with cytochromes P450. Front Pharmacol. 2017:8:159.

20. Pandey AV, Flück CE. NADPH P450 oxidoreductase: structure, function, and pathology of diseases. Pharmacol Ther. 2013;138:229-54.

21. Hughes AL, Powell DW, Bard M, Eckstein J, Barbuch R, Link AJ, et al. Dap1/ PGRMC1 binds and regulates cytochrome P450 enzymes. Cell Metab. 2007:5:143-9.

22. Ahmed ISA, Chamberlain C, Craven RJ. S2R(Pgrmc1): the cytochromerelated sigma-2 receptor that regulates lipid and drug metabolism and hormone signaling. Expert Opin Drug Metab Toxicol. 2012;8:361-70.
23. Wendler A, Wehling M. PGRMC2, a yet uncharacterized protein with potential as tumor suppressor, migration inhibitor, and regulator of cytochrome P450 enzyme activity. Steroids. 2013;78:555-8.

24. Albrecht C, Huck V, Wehling M, Wendler A. In vitro inhibition of SKOV-3 cell migration as a distinctive feature of progesterone receptor membrane component type 2 versus type 1. Steroids. 2012;77:1543-50.

25. Piel RB, Shiferaw MT, Vashisht AA, Marcero JR, Praissman JL, Phillips JD, et al. A novel role for progesterone receptor membrane component 1 (PGRMC1): a partner and regulator of ferrochelatase. Biochemistry. 2016:55:5204-17

26. Cahill MA. The evolutionary appearance of signaling motifs in PGRMC1. Biosci Trends. 2017;11:179-92.

27. Peluso JJ, Griffin D, Liu X, Horne M. Progesterone receptor membrane component-1 (PGRMC1) and PGRMC-2 interact to suppress entry into the cell cycle in spontaneously immortalized rat granulosa cells. Biol Reprod. 2014;91:104.

28. Griffin D, Liu X, Pru C, Pru JK, Peluso JJ. Expression of progesterone receptor membrane component- 2 within the immature rat ovary and its role in regulating mitosis and apoptosis of spontaneously immortalized granulosa cells. Biol Reprod. 2014:91:36.

29. Luciano AM, Lodde V, Franciosi F, Ceciliani F, Peluso JJ. Progesterone receptor membrane component 1 expression and putative function in bovine oocyte maturation, fertilization, and early embryonic development. Reproduction. 2010;140:663-72.

30. Lodde V, Peluso JJ. A novel role for progesterone and progesterone receptor membrane component 1 in regulating spindle microtubule stability during rat and human ovarian cell mitosis. Biol Reprod. 2011;84:715-22.

31. Gupta GD, Coyaud É, Gonçalves J, Mojarad BA, Liu Y, Wu Q, et al. A dynamic protein interaction landscape of the human centrosome-cilium interface. Cell. 2015;163:1484-99.

32. Hanson D, Stevens A, Murray PG, Black GCM, Clayton PE. Identifying biological pathways that underlie primordial short stature using network analysis. J Mol Endocrinol. 2014;52:333-44.

33. Scholey JM, Brust-Mascher I, Mogilner A. Cell division. Nature. 2003:422:746-52

34. Malik R, Lenobel R, Santamaria A, Ries A, Nigg EA, Körner R. Quantitative analysis of the human spindle phosphoproteome at distinct mitotic stages. J Proteome Res. 2009;8:4553-63.

35. McCloy RA, Parker BL, Rogers S, Chaudhuri R, Gayevskiy V, Hoffman NJ, et al. Global phosphoproteomic mapping of early mitotic exit in human cells identifies novel substrate dephosphorylation motifs. Mol Cell Proteomics. 2015;14:2194-212.

36. Kind B, Koehler K, Krumbholz M, Landgraf D, Huebner A. Intracellular ROS level is increased in fibroblasts of triple A syndrome patients. J Mol Med. 2010;88:1233-42.

37. Joglekar AP, Bloom KS, Salmon ED. Mechanisms of force generation by end-on kinetochore-microtubule attachments. Curr Opin Cell Biol. 2010;22:57-67.

38. Booth DG, Hood FE, Prior IA, Royle SJ. A TACC3/ch-TOG/clathrin complex stabilises kinetochore fibres by inter-microtubule bridging. EMBO J. 2011;30:906-19.

39. Ando $\mathrm{S}$, Yang $\mathrm{H}$, Nozaki N, Okazaki T, Yoda K. CENP-A, -B, and -C chromatin complex that contains the I-Type a-satellite array constitutes the prekinetochore in HeLa Cells. Mol Cell Biol. 2002;22:2229-41.

40. Lee $\mathrm{H}-\mathrm{C}$, Yin $\mathrm{P}-\mathrm{H}$, Chi C-W, Wei Y-H. Increase in mitochondrial mass in human fibroblasts under oxidative stress and during replicative cell senescence. J Biomed Sci. 2002;9(6 Pt 1):517-26.

41. Huebner A, Kaindl AM, Braun R, Handschug K. New insights into the molecular basis of the triple A syndrome. Endocr Res. 2002;28:733-9.

42. Jühlen R, Idkowiak J, Taylor AE, Kind B, Arlt W, Huebner A, et al. Role of ALADIN in human adrenocortical cells for oxidative stress response and steroidogenesis. PLoS ONE. 2015;10:e0124582.

43. Marumoto T, Zhang D, Saya H. Aurora-A-a guardian of poles. Nat Rev Cancer. 2005;5:42-50

44. Barr AR, Gergely F. Aurora-A: the maker and breaker of spindle poles. J Cell Sci. 2007;120(Pt 17):2987-96.

45. Meunier S, Vernos I. Microtubule assembly during mitosis-from distinct origins to distinct functions? J Cell Sci. 2012;125:2805-14.

46. Rosenblatt J. Spindle assembly: asters part their separate ways. Nat Cell Biol. 2005:7:219-22. 
47. Khodjakov A, Cole RW, Oakley BR, Rieder CL. Centrosome-independent mitotic spindle formation in vertebrates. Curr Biol. 2000;10:59-67.

48. Mahoney NM, Goshima G, Douglass AD, Vale RD. Making microtubules and mitotic spindles in cells without functional centrosomes. Curr Biol. 2006;16:564-9.

49. Mastronarde DN, McDonald KL, Ding R, McIntosh JR. Interpolar spindle microtubules in PTK cells. J Cell Biol. 1993;123(6 Pt 1):1475-89.

50. Glotzer M. The $3 \mathrm{Ms}$ of central spindle assembly: microtubules, motors and MAPs. Nat Rev Mol Cell Biol. 2009:10:9-20.

51. Vanneste D, Ferreira V, Vernos I. Chromokinesins: localization-dependent functions and regulation during cell division. Biochem Soc Trans. 2011;39:1154-60

52. McEwen BF, Heagle AB, Cassels GO, Buttle KF, Rieder CL. Kinetochore fiber maturation in PtK1 cells and its implications for the mechanisms of chromosome congression and anaphase onset. J Cell Biol. 1997;137:1567-80.

53. Bakhoum SF, Thompson SL, Manning AL, Compton DA. Genome stability is ensured by temporal control of kinetochore-microtubule dynamics. Nat Cell Biol. 2009;11:27-35.
54. Zhai Y, Kronebusch PJ, Borisy GG. Kinetochore microtubule dynamics and the metaphase-anaphase transition. J Cell Biol. 1995;131:721-34.

55. National Center for Biotechnology Information, U.S. National Library of Medicine. Basic Local Alignment Search Tool. Bethesda MD, USA; 2013. https://blast.ncbi.nlm.nih.gov/Blast.cgi.

56. Hlavaty J, Ertl R, Miller I, Gabriel C. Expression of progesterone receptor membrane component 1 (PGRMC1), progestin and adipoQ receptor 7 (PAQPR7), and plasminogen activator inhibitor 1 RNAbinding protein (PAIRBP1) in glioma spheroids in vitro. Biomed Res Int. 2016;2016:8065830

57. R Core Team. R. A language and environment for statistical computing. Vienna, Austria: R Foundation for Statistical Computing; 2017. https:// www.R-project.org/.

58. Mirman D. Growth curve analysis and visualization using R. 1st ed. Boca Raton: Chapman and Hall/CRC; 2014.

59. Mirman D, Dixon JA, Magnuson JS. Statistical and computational models of the visual world paradigm: growth curves and individual differences. J Mem Lang. 2008;59:475-94.
Ready to submit your research? Choose BMC and benefit from:

- fast, convenient online submission

- thorough peer review by experienced researchers in your field

- rapid publication on acceptance

- support for research data, including large and complex data types

- gold Open Access which fosters wider collaboration and increased citations

- maximum visibility for your research: over $100 \mathrm{M}$ website views per year

At BMC, research is always in progress.

Learn more biomedcentral.com/submissions 